\title{
Research on Switching Strategy of Mobile Cloud Computing based on Fusion of Threshold Judgment and Network Selection
}

\author{
Jun Wu and Zhijun Chen \\ College of Mechatronics \& Information, Yiwu Industrial \& Commercial College, \\ Yiwu, Zhejiang, China \\ 78549608@qq.com
}

\begin{abstract}
In order to improve the service performance of mobile cloud computing, the paper strategy launched a study about switching strategy of mobile cloud computing, we propose a fused mobile cloud computing switching method based on threshold judgment and network selection. This method divided the switching strategy of mobile cloud computing process into two steps, first by a threshold judgment to determine whether to switch, followed by the use of multi-attribute decision making method to select the best candidate network for the switching .Experimental results show that the proposed switching method has more excellent switching performance than Saw method and TOPSIS method, at the three indicators of switching frequency, completion time, the energy consumption of the mobile terminal have shown outstanding advantages.
\end{abstract}

Keywords: Mobile cloud computing, Switching strategy, Threshold judgment, Network selection

\section{Introduction}

With the explosive growth of mobile applications and the rise of cloud computing concepts, mobile cloud computing has been considered a potentially important technology of mobile services. Mobile Cloud computing is applied cloud computing to a mobile environment, solving the discussed problem of performance insufficient of the mobile terminal in a mobile computing [1].

In mobile cloud computing, through reasonable and effective computing switching and communication switching, not only can extend the battery life of mobile terminals, improve data storage and processing capacity, reduce the economic cost of computing, but also to improve the utilization of agency resources, make full use of agency resources and so on [2].

In order to improve the switching efficiency in work process of mobile cloud computing, scholars have proposed a number of ways. Yook and others focus on the division of tasks, on the program calls to build a cost figure based on data sharing and computation time for the task, and then use the branch and bound method and heuristic algorithms to achieve switching based on tasks [3]. Yeo based on switching policy of task division, according to the mathematical modeling, the problem is converted to the maximum flow and minimum cut in order to get the optimal switching [4]. Amoretti proposed an adaptive algorithm for computing migration, using java program to build a cost figure, in the figure parameters are set to all necessary memory, and the visit relationship between various types, and then using minimum cut method generate the candidate uninstall scheme, the last according to memory resources and network bandwidth usage, to determine the optimal switching scheme [5]. Matos changes put forward a real-time recognition and tracking system of moving object realized by calculates the unloading, preclude the use dynamic partitioning to adapt changing of resources, so improving the performance of the algorithm [6]. 
Through a large number of research results of previous can be found that, switching of mobile cloud computing to solve two key questions: First, computing when is the best switching time; Second, computing which network to switch are optimal for tasks [7]. In this paper, under the guidance of this idea, building a switching strategy research of fused mobile cloud computing between network selection and threshold judgment, using stage of the threshold judgment to determine the best switching time for computing tasks, using the network selection to determine which network is optimal for switching.

\section{Switching Strategy of Mobile Cloud Computing Proposed in this Paper}

In a variety of mobile cloud computing switching method has appeared, the vertical switching strategy is a relatively mature method. In general, the switching process can be divided into the three phases: cloud collection, switching decision, switching performs, wherein the switching decision is a core phase. In the vertical switching strategy, mobile terminal or network based on system of the discovery phase notified of available network information to perform vertically access network selection algorithm, to determine the switching implementation of the objectives network.

In the mobile communication system vertical switching does not exist the so-called best evaluation criteria, But according to the switching requirements of different users to choose the best program, for example: the maximum price, the best quality of service and the lowest cost of services such as switching strategy. Under different scenarios have different switching strategy, based on QoS guarantee operations, and based on the individual needs of other users and based on job performance optimization? Of course, these policies also apply to mobile computing.

In the mobile computing environment, if only use network performance is better than user's current network as a handover decision criterion, will lead to decreased performance of the system, increased power consumption of mobile terminal, frequent occurrence of switching and other issues. Therefore, need some of the more rational handover decision criteria to judgment when to trigger the switching, improving system performance.

Based on this consideration, in this paper switching work of mobile cloud computing is divided into two phases. First finding to trigger the best time startup node and the switching conditions through the threshold judgment; then select the network to find the most suitable switching networks.

\subsection{Work of Threshold Judgment Stage}

Handover decision algorithm of mobile computing environment, generally to determine whether the current environment achieve switching occurs condition, to consider switching when achieve switching conditions that otherwise continue to run in the original network. If you do not set minimum performance standards, every time testing as long as to find better execution environment, or the current network is no longer available, switching ensues. This means that detection of network parameters is available nearby, switching criteria for deciding whether trigger switching.

This design criterion as follows:

First, if the current network is unavailable, execute switching;

Second, if the current network is available, but the vicinity alternate network meets one of the following two conditions, then switching is performed.

Condition One: All attribute parameter of alternative network must be greater than or equal to the corresponding attribute parameters of current network multiplied by the minimum equivalence factor, as shown in Equation (1).

$$
\forall A_{i}^{S}>\forall A_{i}^{C} \cdot \eta_{i}
$$


The formula, $A_{i}^{s}$ represents the $i$-th attribute parameters of the alternative network, $A_{i}^{S}$ represents the $i$-th attribute parameters of the current network, $\eta_{i}$ denotes the minimum equivalent factor.

Second condition:All attribute parameter of alternative network at least one parameter must be greater than the corresponding attribute parameters of current network multiplied by the minimum improvement factor, as shown in Equation (2).

$$
\hat{A}^{s}>\hat{A}^{c} \cdot \lambda
$$

The formula, $\hat{A}^{s}$ represents parameters of high performance alternative network, $\hat{A}^{c}$ represents the parameter corresponding to the current network, $\lambda$ represents the minimum improvement factor.

In fact the above criterion can be explained as: First, if the current network is unavailable, and bound to select an available network in place of the current network, it is necessary to switch at the time, switched threshold can be set as cut-off value of available and unavailable;

Second, if the current network is still available, but the various performance parameters of alternative network are better than the corresponding parameter of the current network, this time should be switched, each is actually various performance parameters value of the current network.

Third, if the current network is still available, but there is an alternative network of performance parameters particular superior to the corresponding to the parameters of current network, this time should be switched, switched threshold is the value of parameter of current network multiplied by maximum magnification may increased.

\subsection{Work of Network Selection Phase}

After the previous stage of the work, we actually first determines whether have to switch or should be switched. But exactly which network to switch is required by the work of network selection phase to complete.

Network selection algorithm is try to find a network, attribute parameter of the network does not poor than the parameters corresponding to current network, and at least one parameter have significant improvement. To achieve the above comparison, it should meet at the two consecutive detection, the same load on the network is not changed, and the corresponding parameters are fixed values. Accordingly, the scope characterize of parameter values the algorithm using is "performance equal" or "significantly improved performance."

In mobile cloud computing network environment, it may occur to switch between different wireless access technologies and management domains, handover decision is no longer based on a parameter, but on the basis of comprehensive considerate multiple parameters to switch judgment. For example, the service price, power consumption, QoS support. Furthermore, users can choose network according to their favorite. This various judgment metrics must be considered in combination, and sometimes the effect of different metrics opposite to each other, Then you have to weigh of judgment consider various factors. So, this is a typical multi-attribute decision making problems. To this end, switching strategy in this paper constructed the work of the network selection phase to achieve by multi-attribute decision.

Set available alternative network for current network with $m$, Each alternative network $N_{i}$ will need to compare $n$ attributes $A_{j}$. And then determine which alternative network should be the ultimate network replace the current network, you need to consider the importance of all the properties $A_{j}$ for the overall performance of network, To this end, 
each property $A_{j}$ will be provided corresponding to an weighting coefficient $w_{j}$, and the title $w_{j}$ are 1, that satisfies the formula (3).

$$
\sum_{j=1}^{n} w_{j}=1
$$

In this paper, multi-attribute decision making problem solving, by using information entropy in information theory to determine the weight of each network attribute, and then using the composite index of computing network with a simple weighted method, and then the composite index to be sorted. Theory, information entropy has a measure of the degree of systematic differences. If the degree of difference of a network attributes between networks is greater, Information entropy is smaller, the amount of information provided by this attribute are greater; conversely, the smaller the difference of the degree of a property, the greater the information entropy, the amount of information provided by the property is smaller. Therefore, by calculating the information entropy of the properties of network to determine the weight of attributes.

Calculated comprehensive attribute information entropy of alternative network, such as (4) as shown in the equation (4):

$$
E\left(N_{i}\right)=-\sum_{j=1}^{n} A_{i j} \ln A_{i j}
$$

In the formula, $E\left(N_{i}\right)$ represents of the information entropy $N_{i}$ of network .

Accordingly, the difference degree of this network attribute can follow the formula (5) is calculated:

$$
W\left(N_{i}\right)=1-E\left(N_{i}\right)=1+\sum_{j=1}^{n} A_{i j} \ln A_{i j}
$$

Thus, we can obtain the weights of each attribute, as shown in the formula (6).

$$
w_{i}=\frac{W\left(N_{i}\right)}{\sum_{i=1}^{n} W\left(N_{i}\right)}
$$

In this way, we can calculate the multi-attribute evaluation results for each network, as shown in Equation (7) below.

$$
\operatorname{DS}\left(N_{i}\right)=\sum_{j=1}^{n} A_{i j} w_{j}
$$

Finally, the multi-attribute evaluation results were compared to each network, select one of the largest value of network used to replace the current network to switch.

\section{Experiments and Experimental Analysis}

In order to validate advantages and disadvantages of fused switching algorithm based on the threshold judgment and network selection mentioned herein, and related parameters submitted by the task impact on the performance of the algorithm parameters, the paper on matlab7.0 simulated test experiments, and experimental results were analyzed.

\subsection{Experimental Environment Settings}

The simulation experiment is developed based on matlab, simulation program actually consists of three modules: information collection module, handover decision module and 
switching execution module. This article is mainly observed execution performance of decision module, but the information collection module and switching execution module is the secondary part to complete the whole switching process.

Information collection module can be divided into two parts, agency discovery and resource monitoring. In the moving process of the mobile terminal, the agent discovery responsible for discovering the available network around the mobile terminal (including the location of the network, performance, property values, etc.,) and feedback the information to the decision-making module, to facilitate decision-making; resource monitoring is responsible for detecting current status of available networks (performance changes, changes in property values, etc.,) and feedback to the decision module.

Handover decision modules can be divided into two parts: sentencing guidelines and network selection. Sentencing guidelines is responsible for determining whether the available networks agent fund satisfy the requirements to trigger switching, satisfied to trigger then switching otherwise continue execute the original network; Network selection is to choose the best network after the decision criteria for selecting in the available network as the target network to switching. Sentencing guidelines and network selection algorithm is a lot, to choose the best algorithm belongs to the user based on the user's preferences.

Switching execution module can be divided into switching execution, implementation monitoring and the results statistics. Switching execution is responsible for executing the process of switching after selecting the target switching network. Implementation monitoring is monitoring the process of switching implementation for entire task , including executive the task on which agents, the agent's location, task completion statics, task completion time, switching times, etc., and feedback these cases to the results statistical module, result statistics module is responsible for statistics the implementation of the mandate.

\subsection{Experimental Parameter Settings}

In the experiment, there is setted one base station, can access the network twelve and the mobile communication method which can access the network can be used both WLAN and WiFi. Setted the base station in the center position, and the remaining twelve that could access the network is randomly distributed around the base station, setting the base station can cover the range for the $2000 \mathrm{~m}$, the coverage of each wireless network is $200 \mathrm{~m}$ or $300 \mathrm{~m}$. The mobile terminal seeking switching, then do uniform circular motion within the range the base station can covered.

Other parameters are set as shown in Table 1.

Table 1. Experimental Parameter Settings

\begin{tabular}{c|l|l}
\hline No. & \multicolumn{1}{|c}{ Parameter Meaning } & \multicolumn{1}{c}{ Parameter Value } \\
\hline Parameter 1 & Coverage radius of the base station & $2000 \mathrm{~m}$ \\
\hline Parameter 2 & Coverage radius of accessed network & $200 \mathrm{~m}$ or 300m \\
\hline Parameter 3 & The bandwidth of the base station & $100 \mathrm{M}$ \\
\hline Parameter 4 & The bandwidth of accessed network & $2 \mathrm{M}$ \\
\hline Parameter 5 & Transmission power of the base station & $40 \mathrm{dBm}$ \\
\hline Parameter 6 & Transmission power of accessed network & $10 \mathrm{dBm}$ \\
\hline Parameter 7 & Network delay of the base station & $80 \mathrm{~ms}$ \\
\hline Parameter 8 & Network delay of accessed network & $40 \mathrm{~ms}$ \\
\hline Parameter 9 & Computing power of the base station & $200000 \mathrm{MIPS}$ \\
\hline Parameter 10 & Computing power of accessed network & $70000 \mathrm{MIPS}$ \\
\hline Parameter 11 & The size of the task to be executive & $100 \mathrm{MB}$ \\
\hline Parameter 12 & The number of instructions to perform tasks & 2000000000 \\
\hline
\end{tabular}




\subsection{Analysis of Experimental Results}

There are many evaluation criteria for handover algorithm in a mobile communication network, such as: handover success rate, call drop rate, new call blocking rate, the average switching frequency, switching delay and forced interruption rates. These evaluation indexes are not fully applicable to the calculation switching in the mobile computing environment. Evaluation for calculate handover performance parameters used in this section, namely, switching frequency, completion time, power consumption of the mobile terminal.

Switching frequency: Record a computing task from submission to completion totally has gone through many times switching. Of course, reducing the switching frequency is usually preferred starting, frequent switching lead to waste of resources, Bingbing effect in the switching process should be minimized.

Completion time: record total time spent a computing task from submission to completion. Generally, the smaller the better completion time.

Power consumption of the mobile terminal: Records energy consumption at the process of the mobile terminal to complete the task. Generally, the smaller the better energy consumption.

In experiments, in order to compare pros and cons of the performance of this method, also selected TOPSIS method and SAW method, performs switching experiment together, and through the three parameters: the switching frequency, completion time, power consumption of the mobile terminal to obtain the final evaluation.

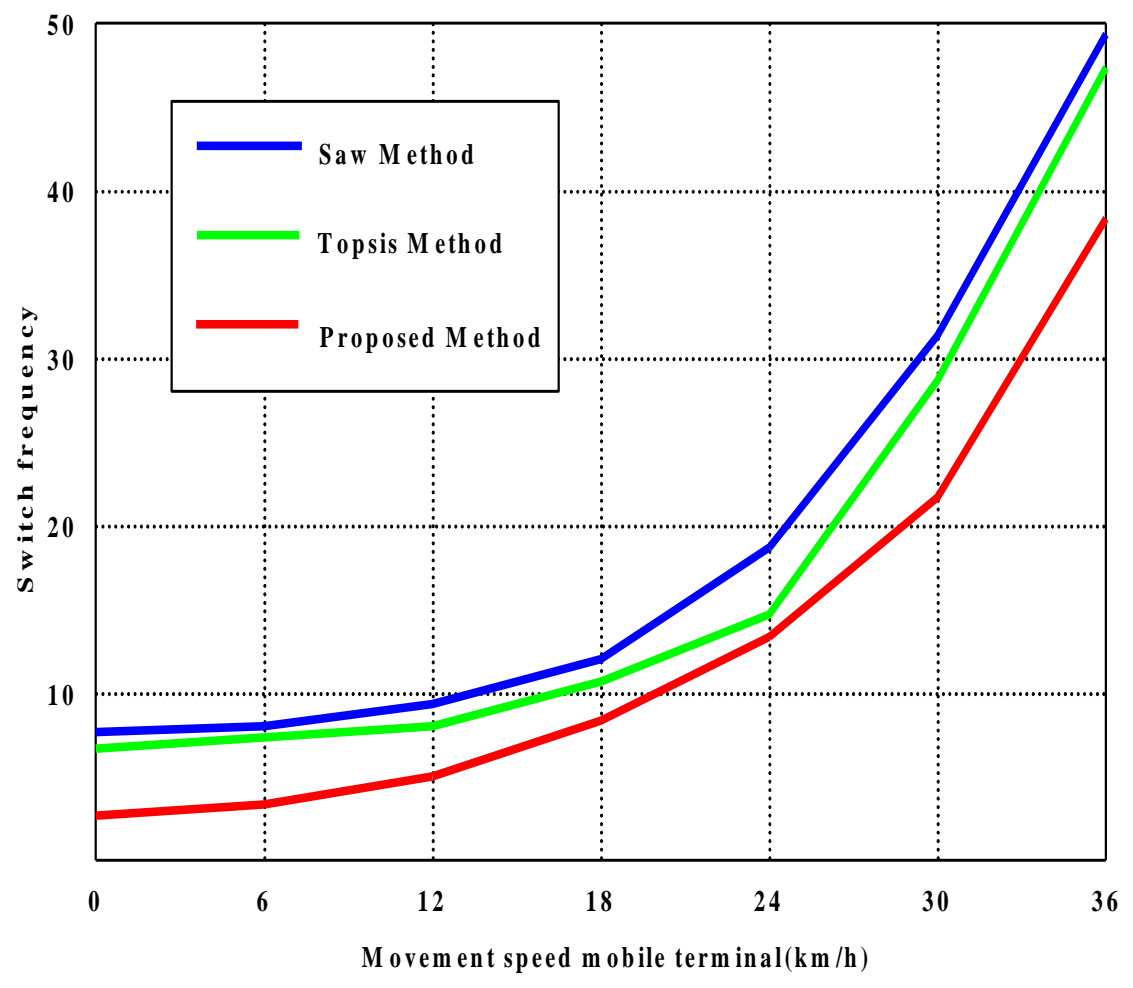

Figure 1. Comparisons of Switching Frequency for Three Methods 


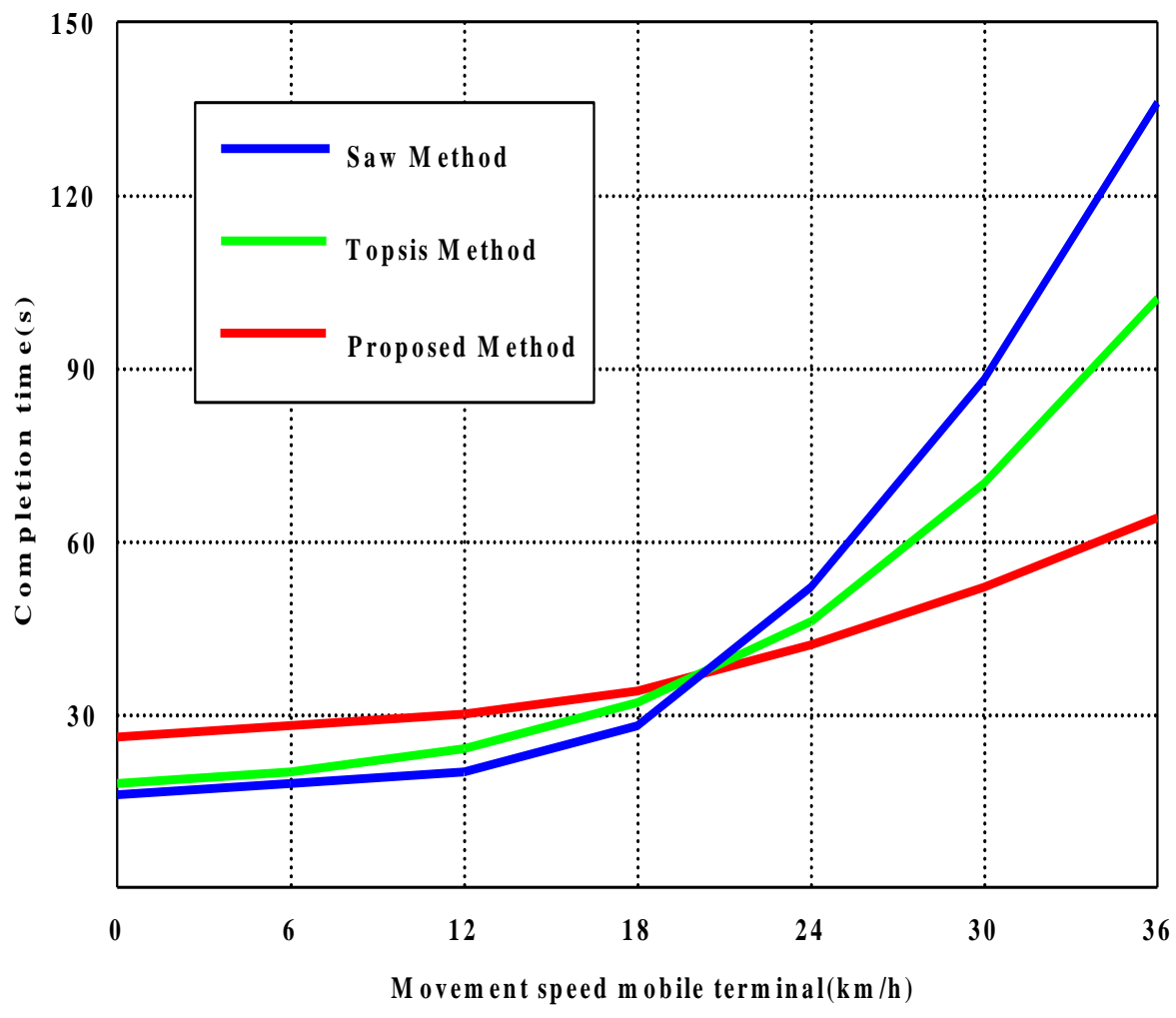

Figure 2. Comparisons of Completion Time for Three Methods

From the curve case shown in Figure 1 and 2, as the moving speed of the mobile terminal accelerating, switching frequency and completion times for the three methods are increasing. On the switching frequency, the moving speed of the mobile terminal after $24 \mathrm{~km} / \mathrm{h}$, the switching frequency of the three methods was significantly increased. But switching frequency of the proposed method is always the least of the three methods. From the switching frequency of the indicators, the proposed method has better switching performance.

In Figure 2, the curve of three method forming a intersection, position occurs when the moving speed of about $20 \mathrm{~km} / \mathrm{h}$, This completion time is a little longer before the intersection of the proposed method, but it is not obvious; After the intersection, the completion time of the proposed method was significantly lower than Topsis methods and Saw methods. Comprehensive view of the entire speed change axis, the proposed method in terms of completion time indicator, also showed better switching performance. 


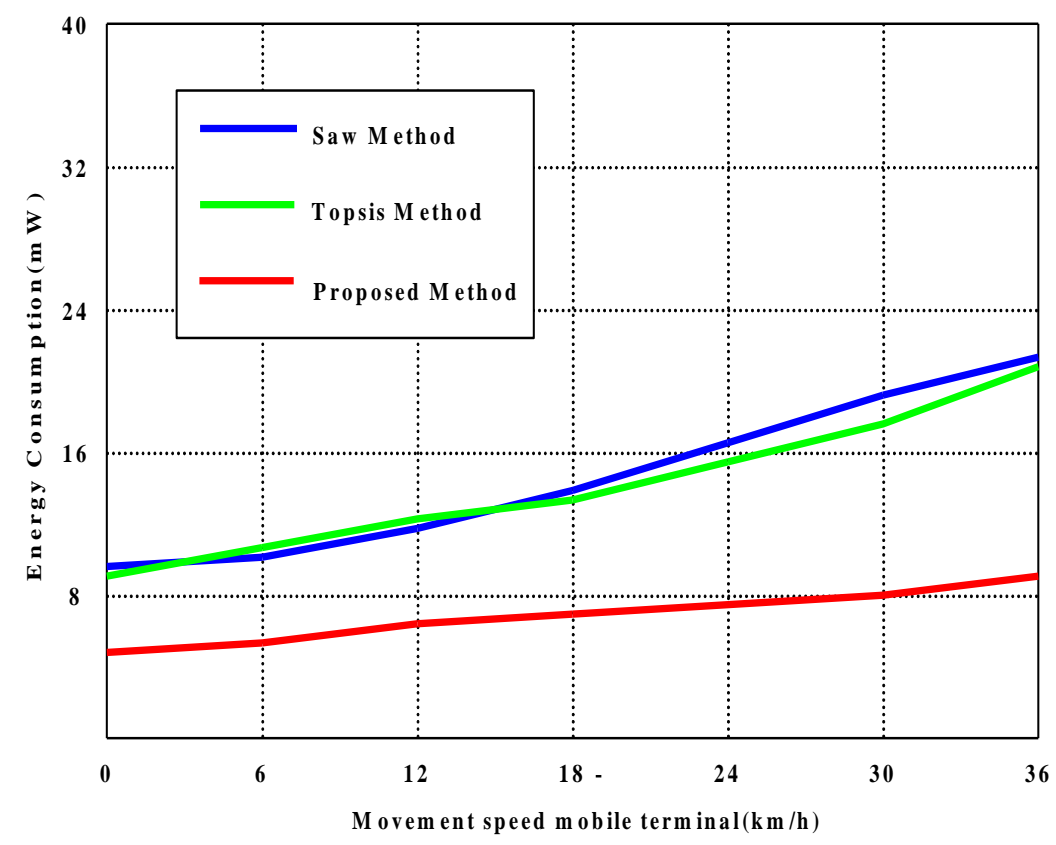

Figure 3. Comparisons of Power Consumption of the Mobile Terminal for Three Methods

As can be seen from Figure 3, power consumption of the intelligent terminal resulting from switching of three methods will increases with speed up of the intelligent terminal moving speed. In contrast, the first two methods with the speed up of moving speed, its smart terminal energy consumption has the trend that increased faster, while the proposed method has remained relatively flat trend, and energy consumption in absolute value of three methods is the lowest. Therefore, from this indicator that power consumption of the mobile terminal point of view, the proposed method also has better switching performance.

\section{Conclusion}

The mobile terminal during using cloud computing, because of its own continuous movement and change of network nodes of cloud computing will lead to the communication link and calculating link continuously switching, purpose of this switching is to ensure that the mobile terminal can always enjoy the most high quality cloud computing services.

In this paper, the realization of mobile cloud computing switching strategy is divided into two phases to achieve: the first stage first need to be determined whether to switching by the threshold value judgments, This paper examines two cases at this stage that advantages of all the attributes of alternative network and absolute dominance of single attribute; The second phase via network to select the final network to execute switching, the paper chosen at this stage multi-attribute decision making method to select the most suitable switching networks.

Furthermore, chosen TOPSIS method and SAW method of cloud computing, compared with switching strategy of fused mobile cloud computing between network selection and threshold judgment, The experimental results showed that: the proposed method compared with the other two methods, at three indicators of switching frequency, completion time and power consumption on the mobile terminal have better switching performance. 


\section{Acknowledgements}

This work is supported by the Program of Scientific and Technological Innovation Personnel of Zhejiang Province (No.2010R30044)

\section{References}

[1] Q. Qi, L. Jianxin and C. Yufei, "Integrated multiple services handoff in mobile cloud computing", Global Information Infrastructure Symposium, vol. 22, (2013), pp. 55-67.

[2] Q. Qi, L. Jianxin and C. Yufei, "Cloud service-aware location update in mobile cloud computing", IET Communications, vol. 8, (2014), pp. 1417-1424.

[3] Y. Juhye and K. Jinsul, "The advanced Korea-computer access assessment system (K-CAAS) on smart mobile cloud environment". Multimedia Tools and Applications, vol. 31, (2014), pp. 128-136.

[4] Y. Hui-Shyong, P. Xiaoshen, L. Hoonjae and L. Hyotaek, "Leveraging client-side storage techniques for enhanced use of multiple consumer cloud storage services on resource constrained mobile devices", Journal of Network and Computer Applications, vol. 43, (2014), pp. 142-156.

[5] A. Michele, G. Alessandro and Z. Francesco, "Towards a formal approach to mobile cloud computing", Euromicro International Conference on Parallel, Distributed, and Network-Based Processing, (2014), pp. 743-750.

[6] M. Rubens and A. Jean, "Sensitivity analysis of a hierarchical model of mobile cloud computing", Simulation Modelling Practice and Theory, vol. 14, (2014), pp. 226-235.

[7] R. M. Reza and R. Jian, "Mobile cloud computing: a survey, state of art and future directions", Mobile Networks and Applications, vol. 19, (2014), pp. 133-143.

[8] G. Abdullah and N. G. Mokatder, "A review on interworking and mobility techniques for seamless connectivity in mobile cloud computing", Journal of Network and Computer Applications, vol. 43, (2014), pp. 84-102.

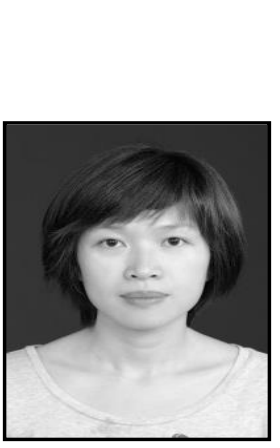

\section{Authors}

Jun Wu, Associate Professor, School of Mechatronics \& Information in Yiwu Industrial \& Commercial College. Born in 1979,Mrs Wu graduated and got master degree from Huazhong University of Science and Techology, and her main research interests are Internet Safety and Cloud Computing.

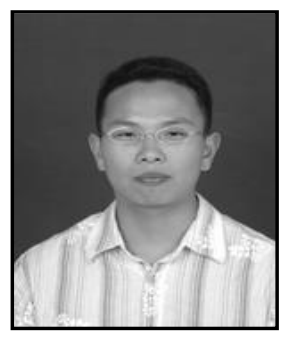

Zhijun Chen, Lecturer, School of Mechatronics \& Information in Yiwu Industrial \& Commercial College. Born in 1982, Mr. Chen graduated and got master degree from Huazhong University of Science and Technology, and his main research interests are Computer Software and Information Technology. 
International Journal of Grid Distribution Computing

Vol.8, No.2 (2015) 